\title{
SOME FIXED POINT THEOREMS FOR NONSELF GENERALIZED CONTRACTION
}

\author{
IOAN A. RUS AND MARCEL-ADRIAN ŞERBAN
}

Received 31 March, 2014

\begin{abstract}
In this paper we give a new proof of a result by S. Reich and A.J. Zaslavski (S. Reich and A.J. Zaslavski, A fixed point theorem for Matkowski contractions, Fixed Point Theory, 8(2007), No. 2, 303-307). Some new fixed point theorems for nonself generalized contractions are also given.
\end{abstract}

2010 Mathematics Subject Classification: 47H10; 54H25

Keywords: metric space, nonself generalized contraction, fixed point, comparison function, maximal displacement functional

\section{INTRODUCTION}

There are many techniques in the fixed point theory of nonself operators (see [10], [4], [6], [9], [19], [20], [2], ... ). An exotic result is given in [14] (see also, [13] and [15]). This result read as follows:

Theorem 1. Let $(X, d)$ be a complete metric space, $Y \subset X$ a nonempty closed subset and $f: Y \rightarrow X$ be a $\varphi$-contraction, where $\varphi$ is a comparison function. We suppose that there exists a bounded sequence $\left(x_{n}\right)_{n \in \mathbb{N}^{*}}$ such that $f^{n}\left(x_{n}\right)$ is defined for all $n \in \mathbb{N}^{*}$. Then $f$ has a unique fixed point $x^{*}$ and $f^{n}\left(x_{n}\right) \rightarrow x^{*}$.

The aim of this paper is to give a new proof of this theorem and to obtain other results of this type.

\section{PRELIMINARIES}

\subsection{Notations}

$\mathbb{N}=\{0,1,2, \ldots\}, \mathbb{N}^{*}=\{1,2,3, \ldots\}$.

$\mathbb{R}_{+}=\{x \in \mathbb{R} \mid x \geq 0\}, \mathbb{R}_{+}^{*}=\{x \in \mathbb{R} \mid x>0\}$

Let $(X, d)$ be a metric space. We will use the following symbols:

$\mathcal{P}(X)=\{Y \mid Y \subset X\}$

This work was supported by a grant of the Romanian National Authority for Scientific Research, CNCS - UEFISCDI, project number PN-II-ID-PCE-2011-3-0094. 
$P(X)=\{Y \subset X \mid Y$ is nonempty $\}, P_{b}(X):=\{Y \in P(X) \mid Y$ is bounded $\}$, $P_{c l}(X):=\{Y \in P(X) \mid Y$ is closed $\}, P_{b, c l}(X):=P_{b}(X) \cap P_{c l}(X)$.

If $f: X \rightarrow X$ is an operator then $F_{f}:=\{x \in X \mid x=f(x)\}$ denotes the fixed point set of the operator $f$. In the case when the operator $f$ has an unique fixed point $x^{*} \in X$ then we write $F_{f}=\left\{x^{*}\right\}$.

The diameter functional $\delta: P(X) \rightarrow \mathbb{R}_{+} \cup\{+\infty\}$ is defined by

$$
\delta(A):=\sup \{d(a, b) \mid a, b \in A\} .
$$

\subsection{Comparison functions}

Let $\varphi: \mathbb{R}_{+} \rightarrow \mathbb{R}_{+}$be a function. We consider the following conditions relative to $\varphi$ :

$\left(\mathrm{i}_{\varphi}\right) \varphi$ is increasing;

(ii $\varphi) \varphi(t)<t, \forall t>0$;

(iii $\varphi) \varphi(0)=0$

$\left.\operatorname{(iv}_{\varphi}\right) \varphi^{n}(t) \rightarrow 0$ as $n \rightarrow \infty, \forall t \in \mathbb{R}_{+}$;

$\left(\mathrm{v}_{\varphi}\right) t-\varphi(t) \rightarrow \infty$ as $t \rightarrow \infty$;

$\left(\operatorname{vi}_{\varphi}\right) \sum_{n=0}^{\infty} \varphi^{n}(t)<+\infty, \forall t \in \mathbb{R}_{+}$.

Definition 1 (I.A. Rus [17]). By definition the function $\varphi$ is a comparison function if it satisfies the conditions $\left(\mathrm{i}_{\varphi}\right)$ and $\left(\mathrm{iv}_{\varphi}\right)$.

Definition 2. A comparison function is:

(a) strict comparison function if it satisfies the condition $\left(\mathrm{v}_{\varphi}\right)$;

(b) strong comparison function if it satisfies the condition $\left(\mathrm{vi}_{\varphi}\right)$.

It is clear that if $\varphi$ is a comparison function then $\varphi(t)<t, \forall t>0$, and $\varphi(0)=0$. If $\varphi$ is a strong comparison function then the functions $\varphi$ and $\sum_{n=0}^{\infty} \varphi^{n}$ are continuous in $t=0$.

For example, if $\varphi(t):=a t, t \in \mathbb{R}_{+}, a \in[0 ; 1[$, then $\varphi$ is a strict and strong comparison function and $\varphi(t):=\frac{t}{1+t}, t \in \mathbb{R}_{+}$, is a strict comparison function which is not a strong comparison function.

Let $\varphi: \mathbb{R}_{+} \rightarrow \mathbb{R}_{+}$be a strict comparison function. In this case we define the function $\theta_{\varphi}: \mathbb{R}_{+} \rightarrow \mathbb{R}_{+}$, defined by,

$$
\theta_{\varphi}(t)=\sup \left\{s \in \mathbb{R}_{+} \mid s-\varphi(s) \leq t\right\} .
$$

We remark that $\theta_{\varphi}$ is increasing and $\theta_{\varphi}(t) \rightarrow 0$ as $t \rightarrow 0$. The function $\theta_{\varphi}$ appears when we study the data dependence of the fixed points.

For more considerations on comparison functions see [17], [1], [21] and [5]. 


\subsection{Maximal displacement functional}

Let $(X, d)$ be a metric space, $Y \in P_{c l}(X)$ and $f: Y \rightarrow X$ be a continuous nonself operator. By the maximal displacement functional corresponding to $f$ we understand the functional $E_{f}: P(Y) \rightarrow \mathbb{R}_{+} \cup\{+\infty\}$ defined by

$$
E_{f}(A):=\sup \{d(x, f(x)) \mid x \in A\} .
$$

We have that:

(i) $A, B \in P(Y), A \subset B$ imply $E_{f}(A) \leq E_{f}(B)$;

(ii) $E_{f}(A)=E_{f}(\bar{A})$ for all $A \in P(Y)$.

Definition 3. An operator $f: Y \rightarrow X$ is $\alpha$-graphic contraction if $0 \leq \alpha<1$ and $x \in Y, f(x) \in Y$ imply

$$
d\left(f^{2}(x), f(x)\right) \leq \alpha d(x, f(x)) .
$$

Example 1. If $f: Y \rightarrow X$ is $\alpha$-contraction then $f$ is $\alpha$-graphic contraction.

Example 2. If $f: Y \rightarrow X$ is $\alpha$-Kannan operator, i.e., $0 \leq \alpha<\frac{1}{2}$, and

$$
d(f(x), f(y)) \leq \alpha[d(x, f(x))+d(y, f(y))], \forall x, y \in Y,
$$

then $f$ is $\frac{\alpha}{1-\alpha}$-graphic contraction.

Also, we have that:

Lemma 1. Let $(X, d)$ be a metric space, $Y \in P_{c l}(X)$ and $f: Y \rightarrow X$ be a continuous $\alpha$-graphic contraction. Then:

(a) $E_{f}(f(A)) \leq \alpha E_{f}(A)$, for all $A \subset Y$ with $f(A) \subset Y$;

(b) $E_{f}(f(A) \cap Y) \leq \alpha E_{f}(A)$, for all $A \subset Y$ with $f(A) \cap Y \neq \varnothing$.

Proof. The proof follows from the definition of $E_{f}$. Let, for example, to prove (b). We have

$$
\begin{aligned}
E_{f}(f(A) \cap Y) & =\sup \{d(x, f(x)) \mid x \in f(A) \cap Y\}= \\
& =\sup \left\{d\left(f(u), f^{2}(u)\right) \mid u \in A, f(u) \in Y\right\} \leq \\
& \leq \alpha \sup \{d(u, f(u)) \mid u \in A\}= \\
& =\alpha E_{f}(A)
\end{aligned}
$$

\subsection{Matrices convergent to 0}

Definition 4. A matrix $S \in \mathbb{R}_{+}^{m \times m}$ is called a matrix convergent to zero iff $S^{k} \rightarrow 0$ as $k \rightarrow+\infty$.

Theorem 2 (see [12], [16], [23], [10]). Let $S \in \mathbb{R}_{+}^{m \times m}$. The following statements are equivalent:

(i) $S$ is a matrix convergent to zero; 
(ii) $S^{k} x \rightarrow 0$ as $k \rightarrow+\infty, \forall x \in \mathbb{R}^{m}$;

(iii) $I_{m}-S$ is non-singular and

$$
\left(I_{m}-S\right)^{-1}=I_{m}+S+S^{2}+\cdots
$$

(iv) $I_{m}-S$ is non-singular and $\left(I_{m}-S\right)^{-1}$ has nonnegative elements;

(v) $\lambda \in \mathbb{C}, \operatorname{det}\left(S-\lambda I_{m}\right)=0$ imply $|\lambda|<1$;

(vi) there exists at least one subordinate matrix norm such that $\|S\|<1$.

The matrices convergent to zero were used by A. I. Perov [11] (see also [10] pp. 432-434) to generalize the contraction principle in the case of generalized metric spaces with the metric taking values in the positive cone of $\mathbb{R}^{m}$.

\section{A NEW PROOF OF THEOREM 1}

Now we present a new proof of Theorem 1. Let $A \in P_{b, c l}(Y)$ be such that $x_{n} \in A$, for all $n \in \mathbb{N}^{*}$. We consider the following standard construction in the fixed point theory for the nonself operators (see for example [8] and [7]).

Let $A_{1}:=\overline{f(A)}, A_{2}:=\overline{f\left(A_{1} \cap A\right)}, \ldots, A_{n+1}:=\overline{f\left(A_{n} \cap A\right)}, n \in \mathbb{N}^{*}$. We remark that:

(a) $A_{n+1} \subset A_{n}, \forall n \in \mathbb{N}^{*}$;

(b) $f^{n}\left(x_{n}\right) \in A_{n}, \forall n \in \mathbb{N}^{*}$, so $A_{n} \neq \varnothing, \forall n \in \mathbb{N}^{*}$.

Since $f$ is a $\varphi$-contraction, i.e., $\varphi: \mathbb{R}_{+} \rightarrow \mathbb{R}_{+}$is a comparison function such that

$$
d(f(x), f(y)) \leq \varphi(d(x, y)), \forall x, y \in Y,
$$

it follows that

$$
\delta(f(B)) \leq \varphi(\delta(B)), \forall B \in P_{b}(Y) .
$$

From the properties of $\varphi$ and $\delta$ we have

$$
\begin{aligned}
\delta\left(A_{n+1}\right) & =\delta\left(\overline{f\left(A_{n} \cap A\right)}\right)=\delta\left(f\left(A_{n} \cap A\right)\right) \leq \delta\left(f\left(A_{n}\right)\right) \leq \\
& \leq \varphi\left(\delta\left(A_{n}\right)\right) \leq \cdots \leq \varphi^{n+1}(\delta(A)) \rightarrow 0
\end{aligned}
$$

as $n \rightarrow+\infty$. From Cantor intersection lemma we have

$$
A_{\infty}:=\bigcap_{n \in \mathbb{N}} A_{n} \neq \varnothing, \delta\left(A_{\infty}\right)=0 \text { and } f\left(A_{\infty} \cap A\right) \subset A_{\infty} .
$$

From $A_{\infty} \neq \varnothing$ and $\delta\left(A_{\infty}\right)=0$, we have that $A_{\infty}=\left\{x^{*}\right\}$. On the other hand $f^{n}\left(x_{n}\right) \in A_{n}$ and $f^{n-1}\left(x_{n}\right) \in A_{n-1} \cap Y$. This implies that $\left\{f^{n}\left(x_{n}\right)\right\}_{n \in \mathbb{N}}$ and $\left\{f^{n-1}\left(x_{n}\right)\right\}_{n \in \mathbb{N}}$ are fundamental sequences. Since $A_{n}, n \in \mathbb{N}^{*}$, are closed, it follows that

$$
f^{n-1}\left(x_{n}\right) \rightarrow x^{*} \text { and } f^{n}\left(x_{n}\right) \rightarrow x^{*} \text { as } n \rightarrow+\infty .
$$

Since $f$ is continuous then $f^{n}\left(x_{n}\right) \rightarrow f\left(x^{*}\right)$, so $f\left(x^{*}\right)=x^{*}$.

With respect to the data dependence of the fixed point, in Theorem 1, we have the following result: 
Theorem 3. Let $f: Y \rightarrow X$ be as in Theorem 1, where $\varphi$ is a strict comparison function. Then:

(a) $d\left(f^{n}\left(x_{n}\right), x^{*}\right) \leq \varphi\left(d\left(x_{n}, x^{*}\right)\right), \forall n \in \mathbb{N}^{*}$;

(b) $d\left(x, x^{*}\right) \leq \theta_{\varphi}(d(x, f(x))), \forall x \in Y$;

(c) Let $g: Y \rightarrow X$ be such that:

(1) there exists $\eta>0$ such that $d(f(x), g(x)) \leq \eta, \forall x \in Y$;

(2) $F_{g} \neq \varnothing$.

Then

$$
d\left(x^{*}, y^{*}\right) \leq \theta_{\varphi}(\eta), \forall y^{*} \in F_{g} .
$$

Proof. Let us prove $(b)$ and $(c)$.

$(b)$. The conclusion $(b)$ follows from the following estimation

$$
d\left(x, x^{*}\right) \leq d(x, f(x))+d\left(f(x), x^{*}\right) \leq d(x, f(x))+\varphi\left(d\left(x, x^{*}\right)\right), \forall x \in Y .
$$

So,

$$
d\left(x, x^{*}\right)-\varphi\left(d\left(x, x^{*}\right)\right) \leq d(x, f(x)), \forall x \in Y .
$$

$(c)$. Let $y^{*} \in F_{g}$ then from $(b)$ it follows

$$
d\left(x^{*}, y^{*}\right) \leq \theta_{\varphi}\left(d\left(y^{*}, f\left(y^{*}\right)\right)\right)=\theta_{\varphi}\left(d\left(g\left(y^{*}\right), f\left(y^{*}\right)\right)\right) \leq \theta_{\varphi}(\eta) .
$$

For more considerations on data dependence of the fixed points for nonself $\varphi$ contractions see [3], [18] and [22].

\section{A FIXED POINT THEOREM FOR NONSELF KANNAN OPERATORS}

We have:

Theorem 4. Let $(X, d)$ be a complete metric space, $Y \subset X$ a nonempty bounded closed subset and $f: Y \rightarrow X$ a continuous operator. We suppose that:

(i) $f$ is $\alpha$-Kannan operator;

(ii) there exists a sequence $\left(x_{n}\right)_{n \in \mathbb{N}^{*}}$ in $Y$ such that $f^{n}\left(x_{n}\right)$ is defined for all $n \in \mathbb{N}^{*}$;

(iii) $E_{f}(Y)<+\infty$.

Then:

(a) $F_{f}=\left\{x^{*}\right\}$

(b) $f^{n-1}\left(x_{n}\right) \rightarrow x^{*}$ and $f^{n}\left(x_{n}\right) \rightarrow x^{*}$ as $n \rightarrow+\infty$;

(c) $d\left(x, x^{*}\right) \leq(1+\alpha) d(x, f(x)), \forall x \in Y$;

(d) $d\left(f^{n-1}\left(x_{n}\right), x^{*}\right) \leq \alpha^{n-1}(1-\alpha)^{1-n}(1+\alpha) d\left(x_{n}, f\left(x_{n}\right)\right), \forall n \in \mathbb{N}^{*}$;

(e) Let $g: Y \rightarrow X$ be such that:

(1) there exists $\eta>0$ such that $d(f(x), g(x)) \leq \eta, \forall x \in Y$;

(2) $F_{g} \neq \varnothing$. 
Then

$$
d\left(x^{*}, y^{*}\right) \leq(1+\alpha) \eta, \forall y^{*} \in F_{g} .
$$

Proof. $(a)+(b)$. Let $Y_{1}:=\overline{f(Y)}, Y_{2}:=\overline{f\left(Y_{1} \cap Y\right)}, \ldots, Y_{n+1}:=\overline{f\left(Y_{n} \cap Y\right)}$, $n \in \mathbb{N}^{*}$. We remark that $Y_{n+1} \subset Y_{n}$ and $f^{n}\left(x_{n}\right) \in Y_{n}$, so $Y_{n} \neq \varnothing, n \in \mathbb{N}^{*}$. Since $f$ is $\alpha$-Kannan operator, from Example 2 and Lemma 1, we have that:

$$
\begin{aligned}
\delta\left(Y_{n+1}\right) & =\delta\left(\overline{f\left(Y_{n} \cap Y\right)}\right)=\delta\left(f\left(Y_{n} \cap Y\right)\right) \leq 2 \alpha \cdot E_{f}\left(Y_{n} \cap Y\right)= \\
& =2 \alpha \cdot E_{f}\left(\overline{f\left(Y_{n-1} \cap Y\right)} \cap Y\right)=2 \alpha \cdot E_{f}\left(f\left(Y_{n-1} \cap Y\right) \cap Y\right) \leq \\
& \leq \frac{2 \alpha^{2}}{1-\alpha} E_{f}\left(Y_{n-1} \cap Y\right) \leq \cdots \leq \frac{2 \alpha^{n+1}}{(1-\alpha)^{n}} E_{f}(Y) \rightarrow 0 \text { as } n \rightarrow+\infty .
\end{aligned}
$$

Now the proof is similar with the proof of Theorem 1.

(c). Let $x \in Y$. From the definition of the Kannan operator we have:

$d\left(x, x^{*}\right) \leq d(x, f(x))+d\left(f(x), x^{*}\right) \leq d(x, f(x))+\alpha d(x, f(x)), \forall x \in Y$.

$(d)$ and $(e)$ follow from $(c)$.

\section{OTHER NONSELF GENERALIZED CONTRACTIONS}

\subsection{Cirić-Reich-Rus operators}

Let $(X, d)$ be a metric space, $Y \in P_{c l}(X)$ and $f: Y \rightarrow X$ be a nonself operator. An operator $f: Y \rightarrow X$ is a Cirić-Reich-Rus operator (see [4], [20], [22], ..) if there exist $a, b \in \mathbb{R}_{+}$with $a+2 b<1$ such that

$$
d(f(x), f(y)) \leq a d(x, y)+b[d(x, f(x))+d(y, f(y))], \forall x, y \in Y .
$$

Lemma 2. Let $(X, d)$ be a metric space, $Y \in P_{c l}(X)$ and $f: Y \rightarrow X$ a nonself Cirić-Reich-Rus operator then $f$ is a nonself $\alpha$-graphic contraction with $\alpha=\frac{a+b}{1-b}$.

Proof. Let $x \in Y$ such that $f(x) \in Y$ then

$$
d\left(f^{2}(x), f(x)\right) \leq a d(f(x), x)+b\left[d\left(f(x), f^{2}(x)\right)+d(x, f(x))\right],
$$

so

$$
d\left(f^{2}(x), f(x)\right) \leq \frac{a+b}{1-b} d(x, f(x)) .
$$

Lemma 3. Let $(X, d)$ be a metric space, $Y \in P_{c l}(X)$ and $f: Y \rightarrow X$ a nonself Cirić-Reich-Rus operator then:

(a) $\delta(f(A) \cap Y) \leq a \delta(A)+2 b E_{f}(A)$, for all $A \subset Y$;

(b) $E_{f}(f(A) \cap Y) \leq \alpha E_{f}(A)$, for all $A \subset Y$, where $\alpha=\frac{a+b}{1-b}$. 
Proof. (a). Let $A \subset Y$ then

$$
\begin{aligned}
\delta(f(A) \cap Y) & =\sup \{d(x, y) \mid x, y \in f(A) \cap Y\}= \\
& =\sup \{d(f(u), f(v)) \mid u, v \in A, f(u), f(v) \in Y\} \leq \\
& \leq a \sup \{d(u, v) \mid u, v \in A\}+2 b \sup \{d(u, f(u)) \mid u \in A\}= \\
& =a \delta(A)+2 b E_{f}(A)
\end{aligned}
$$

(b). The proof follows from Lemma 2 and Lemma 1.

Also, for the next result we need the following lemma

Lemma 4 (Cauchy Lemma, [21]). Let $a_{n}, b_{n} \in \mathbb{R}_{+}, n \in \mathbb{N}$. We suppose that:

(i) $\sum_{k=0}^{\infty} a_{k}<+\infty$;

(ii) $b_{n} \rightarrow 0$ as $n \rightarrow \infty$.

Then

$$
\sum_{k=0}^{n} a_{n-k} b_{k} \rightarrow 0 \text { as } n \rightarrow \infty .
$$

Theorem 5. Let $(X, d)$ be a complete metric space, $Y \subset X$ a nonempty bounded closed subset and $f: Y \rightarrow X$ a continuous operator. We suppose that:

(i) $f$ is Cirić-Reich-Rus operator;

(ii) there exists a sequence $\left(x_{n}\right)_{n \in \mathbb{N}^{*}}$ in $Y$ such that $f^{n}\left(x_{n}\right)$ is defined for all $n \in \mathbb{N}^{*}$

(iii) $E_{f}(Y)<+\infty$.

Then:

(a) $F_{f}=\left\{x^{*}\right\}$

(b) $f^{n-1}\left(x_{n}\right) \rightarrow x^{*}$ and $f^{n}\left(x_{n}\right) \rightarrow x^{*}$ as $n \rightarrow+\infty$;

(c) $d\left(x, x^{*}\right) \leq(1+b)(1-a)^{-1} d(x, f(x)), \forall x \in Y$;

(d) $d\left(f^{n-1}\left(x_{n}\right), x^{*}\right) \leq(1+b)(1-a)^{-1} \alpha^{n-1} d\left(x_{n}, f\left(x_{n}\right)\right), \forall n \in \mathbb{N}^{*}$, where $\alpha=\frac{a+b}{1-b}$.

(e) Let $g: Y \rightarrow X$ be such that:

(1) there exists $\eta>0$ such that $d(f(x), g(x)) \leq \eta, \forall x \in Y$;

(2) $F_{g} \neq \varnothing$.

Then

$$
d\left(x^{*}, y^{*}\right) \leq(1+b)(1-a)^{-1} \eta, \forall y^{*} \in F_{g} .
$$

Proof. $(a)+(b)$. Let $Y_{1}:=\overline{f(Y)}, Y_{2}:=\overline{f\left(Y_{1} \cap Y\right)}, \ldots, Y_{n+1}:=\overline{f\left(Y_{n} \cap Y\right)}$, $n \in \mathbb{N}^{*}$. We remark that $Y_{n+1} \subset Y_{n}$ and $f^{n}\left(x_{n}\right) \in Y_{n}$, so $Y_{n} \neq \varnothing, n \in \mathbb{N}^{*}$. Since $f$ is Ćirić-Reich-Rus operator, from Lemma 3 (a), we have that:

$$
\delta\left(Y_{n+1}\right)=\delta\left(\overline{f\left(Y_{n} \cap Y\right)}\right)=\delta\left(f\left(Y_{n} \cap Y\right)\right) \leq
$$




$$
\begin{aligned}
\leq & a \delta\left(Y_{n} \cap Y\right)+2 b E_{f}\left(Y_{n} \cap Y\right) \leq \\
\leq & a \delta\left(Y_{n}\right)+2 b E_{f}\left(Y_{n} \cap Y\right) \leq \cdots \leq \\
\leq & a^{n+1} \delta(Y)+a^{n} 2 b \cdot E_{f}(Y)+ \\
& +a^{n-1} 2 b \cdot E_{f}\left(Y_{1} \cap Y\right)+\cdots+2 b \cdot E_{f}\left(Y_{n} \cap Y\right) .
\end{aligned}
$$

On the other hand, from Lemma 3 (b) we get

$$
\begin{aligned}
E_{f}\left(Y_{k} \cap Y\right) & =E_{f}\left(\overline{f\left(Y_{n-1} \cap Y\right)} \cap Y\right)=E_{f}\left(f\left(Y_{n-1} \cap Y\right) \cap Y\right) \leq \\
& \leq \alpha E_{f}\left(Y_{k-1} \cap Y\right) \leq \cdots<\alpha^{k} E_{f}(Y), k \in N^{*},
\end{aligned}
$$

where $\alpha=\frac{a+b}{1-b}$. Applying Lemma 4 for $a_{n}=a^{n}$ and $b_{n}=2 b \cdot E_{f}\left(Y_{n} \cap Y\right)$ and we get that

$$
\delta\left(Y_{n}\right) \rightarrow 0 \text { as } n \rightarrow+\infty
$$

and the proof is similar with the proof of Theorem 1 .

(c). Let $x \in Y$. From the definition of the Ćirić-Reich-Rus operator we have:

$$
\begin{aligned}
d\left(x, x^{*}\right) & \leq d(x, f(x))+d\left(f(x), x^{*}\right) \leq \\
& \leq d(x, f(x))+a d\left(x, x^{*}\right)+b d(x, f(x)), \forall x \in Y,
\end{aligned}
$$

so

$(d)$ and $(e)$ follow from $(c)$

$$
d\left(x, x^{*}\right) \leq \frac{1+b}{1-a} d(x, f(x)), \forall x \in Y .
$$

\subsection{Perov operators}

Let $(X, d)$ be a generalized metric space with $d: X \times X \rightarrow \mathbb{R}_{+}^{m}, Y \in P_{c l}(X)$ and $f: Y \rightarrow X$ be a nonself operator. By definition (see [17], [20]) $f: Y \rightarrow X$ is a nonself Perov operator if there exists a matrix convergent to zero $S \in \mathbb{R}_{+}^{m \times m}$ such that

$$
d(f(x), f(y)) \leq S \cdot d(x, y), x, y \in Y .
$$

We have the following fixed point results in the case of nonself Perov operators:

Theorem 6. Let $(X, d)$ be a complete generalized metric space with $d: X \times X \rightarrow$ $\mathbb{R}_{+}^{m}, Y \subset X$ a nonempty bounded closed subset and $f: Y \rightarrow X$ an operator. We suppose that:

(i) $f$ is a Perov operator;

(ii) there exists a sequence $\left(x_{n}\right)_{n \in \mathbb{N}^{*}}$ in $Y$ such that $f^{n}\left(x_{n}\right)$ is defined for all $n \in \mathbb{N}^{*}$.

Then:

(a) $F_{f}=\left\{x^{*}\right\}$;

(b) $f^{n-1}\left(x_{n}\right) \rightarrow x^{*}$ and $f^{n}\left(x_{n}\right) \rightarrow x^{*}$ as $n \rightarrow+\infty$.

(c) $d\left(x, x^{*}\right) \leq\left(I_{m}-S\right)^{-1} d(x, f(x)), \forall x \in Y$; 
(d) $d\left(f^{n}\left(x_{n}\right), x^{*}\right) \leq S^{n} d\left(x_{n}, x^{*}\right), \forall n \in \mathbb{N}^{*}$;

(e) Let $g: Y \rightarrow X$ be such that:

(1) there exists $\eta \in\left(\mathbb{R}_{+}^{*}\right)^{m}$ such that $d(f(x), g(x)) \leq \eta, \forall x \in Y$;

(2) $F_{g} \neq \varnothing$.

Then

$$
d\left(x^{*}, y^{*}\right) \leq\left(I_{m}-S\right)^{-1} \eta, \forall y^{*} \in F_{g} .
$$

Proof. $(a)+(b)$. Let $Y_{1}:=\overline{f(Y)}, Y_{2}:=\overline{f\left(Y_{1} \cap Y\right)}, \ldots, Y_{n+1}:=\overline{f\left(Y_{n} \cap Y\right)}$, $n \in \mathbb{N}^{*}$. We remark that $Y_{n+1} \subset Y_{n}$ and $f^{n}\left(x_{n}\right) \in Y_{n}$, so $Y_{n} \neq \varnothing, n \in \mathbb{N}^{*}$. Since $f$ is a Perov we have:

$$
\begin{aligned}
\delta\left(Y_{n+1}\right) & =\delta\left(\overline{f\left(Y_{n} \cap Y\right)}\right)=\delta\left(f\left(Y_{n} \cap Y\right)\right) \leq S \cdot \delta\left(Y_{n} \cap Y\right)=\leq \\
& \leq S \cdot \delta\left(Y_{n}\right) \leq \cdots \leq S^{n+1} \cdot \delta(Y) \rightarrow 0 \text { as } n \rightarrow+\infty .
\end{aligned}
$$

Now the proof is similar with the proof of Theorem 1.

(c). Let $x \in Y$ then we have:

$$
d\left(x, x^{*}\right) \leq d(x, f(x))+d\left(f(x), x^{*}\right) \leq d(x, f(x))+S d\left(x, x^{*}\right), \forall x \in Y,
$$

so

$$
d\left(x, x^{*}\right) \leq\left(I_{m}-S\right)^{-1} d(x, f(x)), \forall x \in Y .
$$

$(d)$ follows from the definition of the Perov operator and $(e)$ is obtained from $(c)$ for $x:=y^{*} \in F_{g}$.

\section{AN OPEN PROBLEM}

The above considerations give rise to the following problem:

Problem 1. Let $(X, d)$ be a complete metric space, $Y$ a nonempty bounded and closed subset of $X$ and $f: Y \rightarrow X$ a nonself operator. We suppose that there exists a sequence $\left(x_{n}\right)_{n \in \mathbb{N}^{*}}$ such that $f^{n}\left(x_{n}\right)$ is defined for all $n \in \mathbb{N}^{*}$. In which additional conditions on $f$ we have that:

(a) $F_{f} \neq \varnothing$ ?

(b) $F_{f}=\left\{x^{*}\right\}$ ?

\section{REFERENCES}

[1] V. Berinde, Iterative Approximation of Fixed Points, 2nd ed., ser. Lecture Notes in Mathematics, 1912. Berlin: Springer, 2007.

[2] V. Berinde and M. Păcurar, "Fixed point theorems for nonself single-valued almost contractions," Fixed Point Theory, vol. 14, no. 2, pp. 301-312, 2013.

[3] A. Chiş-Novac, R. Precup, and I. A. Rus, "Data dependence of fixed points for nonself generalized contractions," Fixed Point Theory, vol. 10, no. 1, pp. 73-87, 2009.

[4] A. Granas and J. Dugundji, Fixed Point Theory, ser. Springer Monographs in Mathematics. Springer-Verlag, New York, 2003. 
[5] J. Jachymski and I. Jóźwik, "Nonlinear contractive conditions: a comparison and related problems," in Fixed point theory and its applications, ser. Banach Center Publ., vol. 77, doi: 10.4064/bc77-0-10. Polish Acad. Sci., Warsaw, 2007, pp. 123-146. [Online]. Available: http://dx.doi.org/10.4064/bc77-0-10

[6] W. A. Kirk and B. Sims, Eds., Handbook of metric fixed point theory. Kluwer Academic Publishers, Dordrecht, 2001.

[7] R. D. Nussbaum, "The fixed point index and asymptotic fixed point theorems for $K$-setcontractions," Bull. Amer. Math. Soc., vol. 75, pp. 490-495, 1969.

[8] R. D. Nussbaum, The fixed point index and the fixed point theorems for K-set-contractions. ProQuest LLC, Ann Arbor, MI, 1969, thesis (Ph.D.)-The University of Chicago.

[9] D. O'Regan and R. Precup, Theorems of Leray-Schauder type and applications, ser. Series in Mathematical Analysis and Applications. Gordon and Breach Science Publishers, Amsterdam, 2001, vol. 3 .

[10] J. M. Ortega and W. C. Rheinboldt, Iterative solution of nonlinear equations in several variables. Academic Press, New York-London, 1970.

[11] A. I. Perov, "On the Cauchy problem for a system of ordinary differential equations," Pribliz. Metod. Rešen. Dif. Uravnen. Vyp., vol. 2, pp. 115-134, 1964.

[12] R. Precup, "The role of matrices that are convergent to zero in the study of semilinear operator systems," Math. Comput. Modelling, vol. 49, no. 3-4, pp. 703-708, 2009, doi: 10.1016/j.mcm.2008.04.006. [Online]. Available: http://dx.doi.org/10.1016/j.mcm.2008.04.006

[13] D. Reem, S. Reich, and A. J. Zaslavski, "Two results in metric fixed point theory," J. Fixed Point Theory Appl., vol. 1, no. 1, pp. 149-157, 2007, doi: 10.1007/s11784-006-0011-4. [Online]. Available: http://dx.doi.org/10.1007/s11784-006-0011-4

[14] S. Reich and A. J. Zaslavski, "A fixed point theorem for Matkowski contractions," Fixed Point Theory, vol. 8, no. 2, pp. 303-307, 2007.

[15] S. Reich and A. J. Zaslavski, "A note on Rakotch contractions," Fixed Point Theory, vol. 9, no. 1, pp. 267-273, 2008.

[16] I. A. Rus, Principles and Applications of the Fixed Point Theory. Dacia, Cluj-Napoca, 1979.

[17] I. A. Rus, Generalized contractions and applications. Cluj University Press, Cluj-Napoca, 2001.

[18] I. A. Rus, "The theory of a metrical fixed point theorem: theoretical and applicative relevances," Fixed Point Theory, vol. 9, no. 2, pp. 541-559, 2008.

[19] I. A. Rus, "Five open problems in the fixed point theory in terms of fixed point structures (i): singled valued operators," in Fixed Point Theory and Its Applications, R. Espinola, A. Petruşel, and S. Prus, Eds. Cluj-Napoca: House of the Book of Science, 2013, pp. 39-60.

[20] I. A. Rus, A. Petruşel, and G. Petruşel, Fixed point theory. Cluj University Press, Cluj-Napoca, 2008.

[21] I. A. Rus and M.-A. Şerban, "Some generalizations of a Cauchy lemma and applications," in Topics in mathematics, computer science and philosophy. Presa Univ. Clujeană, Cluj-Napoca, 2008, pp. 173-181.

[22] I. A. Rus and M.-A. Şerban, "Basic problems of the metric fixed point theory and the relevance of a metric fixed point theorem," Carpathian J. Math., vol. 29, no. 2, pp. 239-258, 2013.

[23] M.-A. SSerban, "Fixed point theorems on Cartesian product," Fixed Point Theory, vol. 9, no. 1, pp. 331-350, 2008. 
Authors' addresses

Ioan A. Rus

Babeş-Bolyai University of Cluj-Napoca, Department of Mathematics, 1 M. Kogălniceanu, 400084 Cluj-Napoca, Romania

E-mail address: iarus@math.ubbcluj.ro

\section{Marcel-Adrian Şerban}

Babeş-Bolyai University of Cluj-Napoca, Department of Mathematics, 1 M. Kogălniceanu, 400084 Cluj-Napoca, Romania

E-mail address: mserban@math.ubbcluj.ro 